Anaesthesist 2022 $\cdot 71: 526-534$

https://doi.org/10.1007/s00101-021-01073-9

Eingegangen: 14. Januar 2021

Überarbeitet: 11. Juli 2021

Angenommen: 23. Oktober 2021

Online publiziert: 18 . Februar 2022

() Der/die Autor(en) 2022

\section{Auf Nummer sicher? Sicherheitskultur und Patientensicherheit an deutschen anästhesiologischen Kliniken - Eine deutschlandweite Umfrage zum Status quo}

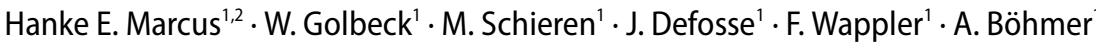 \\ ${ }^{1}$ Klinik für Anästhesiologie und operative Intensivmedizin, Klinikum der Universität Witten/Herdecke, \\ Kliniken der Stadt Köln, Köln, Deutschland \\ ${ }^{2}$ Private Universität Witten/Herdecke gGmbH, Witten, Deutschland
}

\section{Zusatzmaterial online}

Die Online-Version dieses Beitrags (https:// doi.org/10.1007/s00101-021-010739) enthält den dem Artikel zugrunde liegenden Fragebogen.

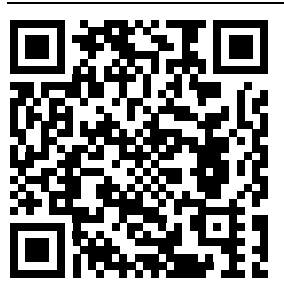

QR-Code scannen \& Beitrag online lesen

\section{Zusammenfassung}

Hintergrund: Zur Erhöhung der Patientensicherheit sind verschiedene Instrumente wie etwa das "Critical Incident Reporting System" (CIRS) oder die Verwendung des „Situation, Background, Assessment, Recommendation (SBAR) System“ entwickelt worden. Ziel war es zu ermitteln, wie Anästhesisten in Deutschland die Sicherheitskultur empfinden, und ob CIRS oder SBAR etabliert sind.

Methodik: Alle registrierten Mitglieder der anästhesiologischen Berufsverbände wurden zu einer Onlineumfrage zum Thema „Patientensicherheit" eingeladen. Es wurden die Themengebiete Sicherheitskultur, CIRS und SBAR erfragt.

Ergebnisse: Die Bewertung der Sicherheitskultur der Anästhesieabteilung war in $76,6 \%$ der Fälle positiv (gesamtes Krankenhaus 54,3\%). Fehlerhafte Verläufe wurden laut $76,5 \%$ offen kommuniziert. $23 \%$ haben den Eindruck, dass bei Fehlern die betreffende Person bloßgestellt würde. Bei einem Drittel finden keine Morbiditäts- und Mortalitäts(M\&M)-Konferenzen statt.

CIRS ist 98,9\% der Befragten bekannt; bei 84,4\% existiert es. CIRS-Meldungen wurden bereits von 54,4\% abgegeben. Regelmäßiges Feedback zu Meldungen erhalten nur $29,4 \%$.

SBAR als Übergabehilfsmittel war 63,7\% unbekannt. $86,1 \%$ halten die Verwendung eines Instruments zur Verbesserung der Übergabequalität für notwendig.

Schlussfolgerungen: Die Qualität der Sicherheitskultur von Anästhesieabteilungen wird positiver bewertet als die des übrigen Krankenhauses. M\&M-Konferenzen sollten größere Verbreitung erfahren.

CIRS ist nahezu allen befragten Anästhesisten bekannt - Feedback erfolgt selten. Es fehlt an Feedbackmechanismen für den Meldenden.

Zur Implementierung von Hilfsmitteln für strukturierte Patientenübergaben sollten in Zukunft mehr Anstrengungen unternommen werden.

\section{Schlüsselwörter}

Patientensicherheit · Sicherheitskultur · Critical Incident Reporting System • „Patient handover" . Umfrage 


\section{Einleitung}

Seit Beginn der Durchführung von Anästhesien hat sich das Risiko anästhesiebedingter Morbidität und Mortalität im Laufe der Jahrzehnte stetig und deutlich vermindert.

Während sich in den 1940er-Jahren noch eine anästhesiebedingte Mortalität in einem von 1000 Fällen ereignet hat, betrug diese in den späten 1980er-Jahren nur noch 0,4/100.000 Anästhesien [4, 15]. Als Gründe für die stetige Reduktion der anästhesiebedingten Mortalität sind u.a. als Meilensteine die Entstehung des Fachgebiets Anästhesie sowie die Einführungen der Pulsoxymetrie und der Kapnometrie zu nennen [24]. Innovationen der Pharmakotherapie und der verwendeten Geräte sowie ein geänderter Anspruch an das vorzuhaltende Personal in Bezug auf Quantität und Qualität sind ebenfalls erhebliche Einflussfaktoren. Es ist kaum quantifizierbar, welchen Anteil ein Wandel der Sicherheitskultur auf die Patientensicherheit genommen hat, zumal bereits der Begriff keine ganz klare und eindeutige Definition besitzt. Eine recht gute Umschreibung des Begriffs Sicherheitskultur ist Guldenmund gelungen: Ihm zufolge ist Sicherheitskultur ein „relativ stabiles, multidimensionales, hypothetisches Konstrukt, das sich auf der Grundlage von geteilten, kulturellen Normen in der Arbeitsumgebung auf Wahrnehmungen und Verhalten der Angestellten und damit auf die Sicherheit in Organisationen auswirkt" [9].

Zu den unterschiedlichen Dimensionen und Teilaspekten einer Sicherheitskultur gehören u.a. eine offene Kommunikation, Teamwork, das Verhalten und die Einstellung der Angestellten, Rückmeldung an Mitarbeiter bzw. institutionalisiertes Feedback, ein sanktionsfreier Umgang mit Fehlern und eine Analyse von unerwünschten Ereignissen, z. B. im Rahmen von Morbiditäts- und Mortalitätskonferenzen und vieles mehr $[1,5,18,19]$.

Sicherheitskultur - subjektive Beurteilung und Erfüllung von Forderungen des Gesetzgebers

Es ist wenig darüber bekannt, wie Anästhesisten in Deutschland die Sicherheitskultur in ihren jeweiligen Abteilungen und Kliniken bewerten, und ob wichtige Bestandteile einer Sicherheitskultur, wie etwa Morbiditäts- und Mortalitätskonferenzen oder das offene und sanktionsfreie Kommunizieren über Fehler, flächendeckend etabliert sind. Ebenso wenig ist bekannt, inwiefern einzelne Maßnahmen zur Steigerung der Patientensicherheit die z. T. sogar vom Gesetzgeber gefordert sind, in Krankenhäusern in anästhesiologischen Abteilungen bzw. Krankenhäusern in Deutschland zum Einsatz kommen [8]. Das Vorhalten eines Risikomanagement- und Fehlermeldesystems (wie etwa das Critical Incident Reporting System (CIRS)) in Krankenhäusern ist eine Forderung des Gemeinsamen Bundesausschuss und Bestandteil des Sozialgesetzbuchs ( $§ 137$ Absatz 1d SGB V) und hat somit Gesetzescharakter. Es ist Pflicht der Krankenhäuser, darüber in den Qualitätsberichten zu informieren. Der Gemeinsame Bundesausschuss hat geregelt, welche Mindestanforderungen an das Fehlermeldesystem gestellt sind. Dazu gehören z.B. Anonymität und Vertraulichkeit, Sanktionsfreiheit, Unabhängigkeit, eine regelmäßige Schulung der Mitarbeiter, eine aktive Unterstützung durch die Führungskräfte, eine Dokumentation, die Nachvollziehbarkeit der Maßnahmen u.v.m. [2, 6, 7, $10]$. Es ist bisher nicht systematisch untersucht, ob die Forderungen des Gemeinsamen Bundesausschuss nach einem Fehlermeldesystem flächendeckend umgesetzt sind.

US-amerikanischen Daten zufolge ereignen sich bis zu $65 \%$ der fehlerhaften Verläufe im medizinischen Bereich aufgrund von ungenügender Kommunikation [22].

Im perioperativen Bereich ist der Aufwachraum aufgrund seiner hohen Dichte an parallelen Ereignissen, wie Neuaufnahmen, Verlegungen, Behandlung von postoperativen Problemen und (Fehl-)Alarmierungen, besonders anfällig für Kommunikationsdefizite. Dieses Problem kann sich durch erhöhten Zeitdruck und einen zu hohen Geräuschpegel aggravieren [3]. Ein Patient durchläuft perioperativ durchschnittlich 2 bis 3 Übergaben von einem Behandlungsteam zum darauf folgenden. Um die Qualität der Vielzahl der perioperativen Übergaben im nichtstörungsfreien Umfeld zu verbessern, empfiehlt die DGAI auf Beschluss des engeren Präsidiums seit 2015 die Verwendung des „Situation, Background, Assessment, Recommendation (SBAR) System". Dieses System zielt darauf $a b$, für Patientenübergaben ein bestimmtes Ablaufschema zu verwenden, und beinhaltet ein störungs- und unterbrechungsfreies Übergabegespräch [3].

Auch hier ist bisher unbekannt, ob das SBAR-System bereits Einzug in den klinischen Alltag gefunden hat.

Das Ziel der vorliegenden Arbeit ist daher, die Einschätzung der Mitglieder der DGAI oder des BDA über die Sicherheitskultur an ihrem Arbeitsplatz sowie den Grad der Etablierung von anerkannten und vom Gesetzesgeber geforderten Methoden zur Erhöhung der Patientensicherheit, wie CIRS und SBAR, systematisch zu erfassen und die Einstellung zu diesen Maßnahmen zu ermitteln.

\section{Methode}

Die vorliegende Untersuchung ist Teil einer umfangreichen Umfrage rund um das Thema "Patientensicherheit". Die hier vorgestellten Ergebnisse betreffen die Themen "Sicherheitskultur", ,"CIRS" und "SBAR". Weitere Themen des Fragebogens (Zusatzmaterial online, s. Box am Anfang des Beitrags) betrafen den Anästhesieausweis [17] sowie die Sicherheitscheckliste.

Der Fragebogen wurde als Link an alle mit einer E-Mail-Adresse bei der DGAI oder beim BDA registrierten Mitglieder ( $n=19.042)$ im Februar 2017 mit der Bitte um Teilnahme an der Umfrage versendet. Alle bei der DGAI oder beim BDA registrierten Mitglieder wurden einmal angeschrieben.

Über einen Zeitraum von 4 Wochen hatten die Mitglieder Gelegenheit, über das Onlineumfragesystem LimeSurvey® (Fa. LimeSurvey $\mathrm{GmbH}$, Hamburg) den Fragebogen zu bearbeiten. Der Fragebogen umfasste neben den Fragen zum Thema Sicherheitskultur, CIRS und SBAR auch allgemeine Daten zu Position, Weiterbildungsstand und Tätigkeitsort der Umfrageteilnehmer. Weitere Fragen befassten sich mit dem Thema Sicherheitscheckliste und Anästhesieausweis [17].

Neben der Beantwortung von Fragen anhand von 6-fach abgestuften Likert-Or- 
Tab. 1 Allgemeine Charakteristika der Umfrageteilnehmer

\begin{tabular}{|l|l|l|l|}
\hline Tab. 1 Allgemeine Charakteristika der Umfrageteilnehmer \\
\hline \multirow{3}{*}{$\begin{array}{l}\text { Position } \\
\text { (Mehrfach- } \\
\text { nennung } \\
\text { möglich) }\end{array}$} & - & $n$ & $\%$ \\
\cline { 2 - 4 } & Chefarzt & 276 & 12,5 \\
\cline { 2 - 4 } & Oberarzt & 721 & 32,7 \\
\cline { 2 - 4 } & Facharzt & 687 & 31,1 \\
\cline { 2 - 4 } & Assistenzarzt & 339 & 15,4 \\
\cline { 2 - 4 } & Ambulante Tätigkeit & 178 & 8,1 \\
\hline \multirow{4}{*}{$\begin{array}{l}\text { Versorgungs- } \\
\text { stufe }\end{array}$} & Universitätsklinikum & 339 & 15,4 \\
\cline { 2 - 4 } & Überregionale Versorgung & 348 & 15,8 \\
\cline { 2 - 4 } & Schwerpunktversorgung & 433 & 19,6 \\
\cline { 2 - 4 } & Grund-/Regelversorgung & 681 & 30,8 \\
\cline { 2 - 4 } & Spezialklinik & 138 & 6,3 \\
\cline { 2 - 4 } & Praxisklinik & 129 & 5,8 \\
\cline { 2 - 4 } & Keine Angabe & 140 & 6,3 \\
\hline
\end{tabular}

\begin{tabular}{|l|l|l|}
\hline Tab. 2 Einschätzung der Fehlerkultur in eigener Anästhesieabteilung \\
\hline $\begin{array}{l}\text { In meiner Anästhesieabteilung herrscht eine gute Fehlerkul- } \\
\text { tur }\end{array}$ & Häufigkeit $n$ & $\begin{array}{l}\text { Gültige } \\
\text { Prozente }\end{array}$ \\
\hline $1 \hat{=}$ Trifft voll und ganz zu & 285 & 15,9 \\
\hline 2 & 599 & 33,4 \\
\hline 3 & 488 & 27,2 \\
\hline 4 & 196 & 10,9 \\
\hline 5 & 118 & 6,6 \\
\hline $6 \hat{=}$ Trifft gar nicht zu & 105 & 5,9 \\
\hline Gesamt & 1791 & 100 \\
\hline
\end{tabular}

Tab. 3 Einschätzung der Fehlerkultur in eigenem Krankenhaus In meinem Krankenhaus herrscht i. Allg. eine gute Fehlerkultur

\begin{tabular}{|l|l|l|}
\hline $1 \hat{=}$ Trifft voll und ganz zu & 85 & 4,9 \\
\hline 2 & 282 & 16,1 \\
\hline 3 & 582 & 33,3 \\
\hline 4 & 401 & 22,9 \\
\hline 5 & 252 & 14,4 \\
\hline $6 \hat{=}$ Trifft gar nicht zu & 146 & 8,4 \\
\hline Gesamt & 1748 & 100 \\
\hline
\end{tabular}

\begin{tabular}{|l|l|l|}
\hline Tab. 4 Bewertung der Kommunikation im Falle von Fehlern - Likertskala \\
\hline $\begin{array}{l}\text { Über fehlerhafte Verläufe wird zu Lernzwecken innerhalb } \\
\text { meiner Abteilung offen kommuniziert }\end{array}$ & Häufigkeit $n$ & $\begin{array}{l}\text { Gültige } \\
\text { Prozente }\end{array}$ \\
\hline 1 = Trifft voll und ganz zu & 432 & 24,4 \\
\hline 2 & 547 & 30,9 \\
\hline 3 & 378 & 21,3 \\
\hline 4 & 197 & 11,1 \\
\hline 5 & 127 & 7,2 \\
\hline $6 \hat{=}$ Trifft gar nicht zu & 92 & 5,2 \\
\hline Gesamt & 1773 & 100 \\
\hline
\end{tabular}

dinalskalen (1: „trifft voll zu“ bis 6: „trifft gar nicht zu“) bestand die Möglichkeit für kategoriale Mehrfachauswahlen. Aus Gründen der vereinfachten Darstellung („Ja“ oder "Nein") wurde bei einigen Fragen die Skala dichotomisiert: Stufen 1-3: "trifft zu“, Stufen 3-6 „trifft nicht zu“ [11].

Die angegebenen Prozentzahlen beziehen sich jeweils, wenn nicht anders dargestellt, auf die stets unterschiedliche Anzahl der Antwortenden einer jeden Frage. Die statistische Auswertung erfolgte mit IBM SPSS Statistics 24 (Fa. IBM Corp., USA). Die Ergebnisse der deskriptiven Statistik werden als absolute und relative Häufigkeiten dargestellt.

Ein Ethikvotum war nicht erforderlich. Die Autoren erklären, dass es keinen Interessenkonflikt in Zusammenhang mit dieser Untersuchung gibt.

\section{Ergebnisse}

Insgesamt wurde die Umfrage von 2208 Teilnehmern bearbeitet. Dies entspricht $11,6 \%$ aller per E-Mail kontaktierten Mitglieder der DGAI bzw. des BDA.

Am Umfrageabschnitt "Sicherheitskultur" beteiligten sich maximal 1791 Teilnehmer $(9,4 \%$ des angeschriebenen Kollektivs), am Umfrageabschnitt "CIRS“ 1821 (9,6\%) Teilnehmer und am Umfrageabschnitt "SBAR“ 1853 Teilnehmer (9,7\%). (Teilnehmer beantworteten nicht alle Fragen.)

Die allgemeinen Charakteristika der Umfrageteilnehmer illustriert • Tab. 1.

\section{Sicherheitskultur}

Die Einschätzung, ob eine gute Fehlerkultur in der eigenen Anästhesieabteilung vorliegt, zeigt - Tab. 2 und - Tab. 3, wie sie allgemein für das gesamte Krankenhaus eingeschätzt wird.

Bei vereinfachter Darstellung und Dichotomisierung der 6-stufigen Likert-Skala ergibt sich das Bild, dass ein Großteil der Befragten ( $n=1372,76,6 \%)$ angab oder größtenteils zustimmte, dass in der Anästhesieabteilung eine gute Fehlerkultur herrsche. Für das gesamte Krankenhaus wurde die Fehlerkultur von 54,3\% $(n=949)$ der Befragten als positiv bewertet. 


\begin{tabular}{|c|c|}
\hline $\begin{array}{l}\text { Über fehlerhafte Verläufe wird zu Lernzwecken innerhalb } \\
\text { meiner Abteilung offen kommuniziert - dichotomisiert }\end{array}$ & Gültige Ja-Prozente \\
\hline Chefarzt & $98,4 \%(n=241)$ \\
\hline Oberarzt & $81,0 \%(n=490)$ \\
\hline Facharzt & $66,0 \%(n=377)$ \\
\hline Assistenzarzt & $64,2 \%(n=174)$ \\
\hline
\end{tabular}

\begin{tabular}{|l|l|l|}
\hline Tab. 6 Bloßstellen bei Fehlern & Häufigkeit $n$ & $\begin{array}{l}\text { Gültige } \\
\text { Prozente }\end{array}$ \\
\hline $\begin{array}{l}\text { Wenn innerhalb meiner Abteilung jemandem ein Fehler } \\
\text { unterläuft, wird diese Person bloßgestellt }\end{array}$ & 47 & 2,7 \\
\hline 1 = Trifft voll und ganz zu & 115 & 6,5 \\
\hline 2 & 246 & 13,9 \\
\hline 3 & 203 & 11,4 \\
\hline 4 & 483 & 27,2 \\
\hline 5 & 679 & 38,3 \\
\hline $6 \hat{=}$ Trifft gar nicht zu & 1773 & 100 \\
\hline Gesamt & & \\
\hline
\end{tabular}

\begin{tabular}{|l|l|}
\hline Tab. 7 Bloßstellen bei Fehlern dichotomisiert nach Hierarchiestufe \\
\hline $\begin{array}{l}\text { Wenn innerhalb meiner Abteilung jemandem ein Fehler } \\
\text { unterläuft, wird diese Person bloßgestellt - dichotomisiert }\end{array}$ & Gültige Ja-Prozente \\
\hline Chefarzt & $3,7 \%(n=9)$ \\
\hline Oberarzt & $19,8 \%(n=121)$ \\
\hline Facharzt & $33,9 \%(n=193)$ \\
\hline Assistenzarzt & $28,5 \%(n=77)$ \\
\hline
\end{tabular}

\begin{tabular}{|l|l|l|}
\hline Tab. 8 Umgang mit Fehlern - Selbsteinschätzung \\
\hline $\begin{array}{l}\text { Wenn ich einen Fehler begehe, behalte ich dies lieber für } \\
\text { mich }\end{array}$ & Häufigkeit $n$ & $\begin{array}{l}\text { Gültige } \\
\text { Prozente }\end{array}$ \\
\hline $1 \hat{=}$ Trifft voll und ganz zu & 18 & 1,0 \\
\hline 2 & 129 & 7,3 \\
\hline 3 & 234 & 13,2 \\
\hline 4 & 226 & 12,7 \\
\hline 5 & 550 & 31,0 \\
\hline $6 \hat{=}$ Trifft gar nicht zu & 617 & 34,8 \\
\hline Gesamt & 1774 & 100 \\
\hline
\end{tabular}

\begin{tabular}{|l|l|}
\hline Tab. $9 \quad$ Umgang mit Fehlern - Selbsteinschätzung der unterschiedlichen Hierarchiestufen \\
\hline $\begin{array}{l}\text { Wenn ich einen Fehler begehe, behalte ich dies lieber für } \\
\text { mich - dichotomisiert }\end{array}$ & Gültige Ja-Prozente \\
\hline Chefarzt & $11,2 \%(n=27)$ \\
\hline Oberarzt & $19,1 \%(n=121)$ \\
\hline Facharzt & $27,3 \%(n=156)$ \\
\hline Assistenzarzt & $28,5 \%(n=77)$ \\
\hline
\end{tabular}

Die überwiegende Mehrzahl der Befragten $(n=1357,76,5 \%)$ gab an, dass über fehlerhafte Verläufe zu Lernzwecken innerhalb der Abteilung offen kommuniziert würde (• Tab. 4).
Die prozentualen Unterschiede der unterschiedlichen Hierarchiestufen zu dieser Frage im Krankenhaus zeigt - Tab. 5.

Die - Tab. 6 zeigt, wie die antwortenden Teilnehmer die Frage bewerteten, ob im Falle eines unterlaufenen Fehlers diese
Person bloßgestellt würde. Bei der vereinfachten dichotomisierten Darstellung wurde dies von $23,1 \%(n=408)$ der Teilnehmer bejaht.

Die Bewertung der unterschiedlichen Hierarchieebenen in Bezug auf die Frage „Wenn innerhalb meiner Abteilung jemandem ein Fehler unterläuft, wird diese Person bloßgestellt" zeigt • Tab. 7.

Umgang mit Fehlern: Die - Tab. 8 illustriert, wie häufig ein Fehler nicht an andere kommuniziert, "für sich behalten wird":

Die Bewertung der unterschiedlichen Hierarchieebenen in Bezug auf diese Frage zeigt • Tab. 9.

Vereinfacht und dichotomisiert dargestellt gaben im Falle eines begangenen Fehlers insgesamt $21,5 \%(n=381)$ der Teilnehmer an, diesen lieber für sich zu behalten.

Im Falle eines persönlich begangenen Fehlers gaben $52,0 \%(n=921)$ der Befragten an, Sorge vor rechtlichen Konsequenzen zu haben. Die überwiegende Mehrzahl ( $n=1451,81,8 \%$ ) gab an, dass die Patientensicherheit im Krankenhaus ausreichend gewährleistet sei.

Morbiditäts- und Mortalitätskonferenzen, in denen kritische Patientenverläufe aufgearbeitet werden, fanden laut den Teilnehmern in den Anästhesieabteilungen von $63,4 \%(n=1089)$ der Befragten statt.

\section{Critical Incident Reporting System}

Der Bekanntheitsgrad des Akronyms CIRS bei den Teilnehmern ist sehr hoch ( $n=1801,98,9 \%)$. In der überwiegenden Mehrzahl ( $n=1544,84,4 \%$ ) der Anästhesieabteilungen ist laut Teilnehmern ein CIRS etabliert.

Die Einschätzung der Teilnehmer bezüglich der Intensität der Nutzung des CIRS zeigt ๑ Tab. 10.

Fast alle Teilnehmer $(n=1494,99,3 \%)$ gaben an, dass jedem Mitarbeiter erlaubt ist, eine CIRS-Meldung abzugeben. In 10 Fällen $(0,5 \%)$ war dies nicht der Fall.

$92,0 \%(n=1357)$ gaben an, dass CIRSMeldungen in der Regel anonym bleiben.

Wer der erste Empfänger einer CIRSMeldung ist, illustriert ๑ Tab. 11.

Mehr als die Hälfte der Antwortenden ( $n=839,54,4 \%$ ) hat bereits eine CIRSMeldung abgegeben. Nur eine Minderheit 
Tab. 10 Einschätzung der Intensität der Nutzung des CIRS

\begin{tabular}{|l|l|l|}
\hline & $\begin{array}{l}\text { Häufig- } \\
\text { keit } n\end{array}$ & Prozent \\
\hline $1 \hat{=}$ sehr intensiv & 82 & 5,7 \\
\hline 2 & 286 & 19,8 \\
\hline 3 & 522 & 36,2 \\
\hline 4 & 261 & 18,1 \\
\hline 5 & 173 & 12,0 \\
\hline $6 \hat{=}$ gar nicht & 119 & 8,2 \\
\hline Gesamt & 1443 & 100 \\
\hline
\end{tabular}

Tab. 12 Bewertung der antwortenden Teilnehmer zu unterschiedlichen Fragen zum Thema CIRS

\begin{tabular}{|l|l|l|}
\hline & $\begin{array}{l}\text { "Ja“ Häu- } \\
\text { figkeit } n\end{array}$ & $\begin{array}{l}\text { Prozent (bezogen auf } \\
\text { 1573 Teilnehmer, die } \\
\text { diesen Fragekomplex } \\
\text { beantwortet haben) }\end{array}$ \\
\hline Ich habe schon aus gemeldeten CIRS-Fällen gelernt & 1012 & 64,3 \\
\hline $\begin{array}{l}\text { Ich habe schon erlebt, dass aus einer CIRS-Meldung } \\
\text { Konsequenzen gezogen wurden }\end{array}$ & 1058 & 67,3 \\
\hline $\begin{array}{l}\text { Die Patientensicherheit konnte durch CIRS in meinem } \\
\text { Krankenhaus verbessert werden }\end{array}$ & 712 & 45,3 \\
\hline $\begin{array}{l}\text { Die Mitarbeitersicherheit konnte durch CIRS in meinem } \\
\text { Krankenhaus verbessert werden }\end{array}$ & 393 & 24,9 \\
\hline $\begin{array}{l}\text { In meiner Abteilung gibt es regelmäßig Feedback be- } \\
\text { züglich eingegangener CIRS-Meldungen }\end{array}$ & 462 & 29,4 \\
\hline
\end{tabular}

( $n=251,11,4 \%)$ glaubt, dass dem CIRS i. Allg. misstraut wird.

Die Bewertung der antwortenden Teilnehmer zu unterschiedlichen Fragen zum Thema CIRS illustriert • Tab. 12.

Die überwiegende Mehrheit der Befragten $(95,4 \%, n=1287)$ hält das CIRS für sinnvoll, um die Patientensicherheit zu erhöhen.

Eine Minderheit von 19\% $(n=338)$ misstraut dem CIRS, weil es eine Möglichkeit bietet, Fehler von Mitarbeitern herauszufinden.

Das Vorhandensein von CIRS in Abhängigkeit von der Versorgungsstufe des Krankenhauses zeigt $\bullet$ Abb. 1.

\section{Situation, Background, Assessment,} Recommendation System

Das Akronym SBAR als Hilfsmittel einer verbesserten Patientenübergabe (z.B. Übergabe vom OP-Anästhesisten an das Aufwachraumpersonal) ist nur einer Minderheit der Teilnehmer $(n=672,30,4 \%)$ bekannt. Noch weniger Befragte gaben an ( $n=176,8 \%$ ), dass SBAR als Hilfsmittel verwendet wird. Hingegen berichten $23,4 \%$
( $n=516)$ der Teilnehmer, dass andere bzw. zusätzliche Hilfsmittel verwendet werden, um vollständige und strukturierte Patientenübergaben zu gewährleisten. Die Notwendigkeit der Verwendung eines Hilfsmittels zur Verbesserung der Übergabequalität sehen $86,1 \%(n=1554)$ der Teilnehmer der Umfrage.

Im praktischen Arbeitsalltag achten $71,7 \%(n=1295)$ der befragten Teilnehmer darauf, dass die Patientenübergabe erst dann begonnen wird, wenn der Patient vollständig an das Monitoring angeschlossen ist, sodass alle zuhören können, während allerdings auch 84,0\% ( $n=1531)$ beobachten, dass währenddessen noch Handlungen am Patienten durchgeführt werden.

$77,5 \%(n=819)$ der Befragten würden sich ein Hilfsmittel oder eine bestimmte Struktur wünschen, um die eigene Patientenübergabe zu verbessern.

\section{Diskussion}

In der vorliegenden Arbeit wurde erstmals eine Einschätzung der Mitglieder der DGAI oder des BDA über die Sicherheitskultur
Tab. 11 Erstempfänger einer CIRS-Meldung

\begin{tabular}{|l|l|l|}
\hline $\begin{array}{l}\text { Erstempfänger einer CIRS-Mel- } \\
\text { dung ist }\end{array}$ & $\begin{array}{l}\text { Häufig- } \\
\text { keit } n\end{array}$ & $\begin{array}{l}\text { Prozent (bezogen auf 1573 Teilnehmer, die } \\
\text { diesen Fragenkomplex beantwortet haben) }\end{array}$ \\
\hline $\begin{array}{l}\text { CIRS-Beauftragter, Anästhesie- } \\
\text { abteilung }\end{array}$ & 414 & 26,3 \\
\hline Chefarzt/-ärztin, Anästhesie & 93 & 5,9 \\
\hline Übergeordneter CIRS-Beauftragter & 660 & 41,9 \\
\hline $\begin{array}{l}\text { Unbekannt, wer CIRS-Meldung } \\
\text { erhält }\end{array}$ & 406 & 25,8 \\
\hline
\end{tabular}

an ihrem Arbeitsplatz sowie den Grad der Etablierung von anerkannten Methoden zur Erhöhung der Patientensicherheit, wie CIRS und SBAR, erfasst. Für die Interpretation der Ergebnisse ist im Hinblick auf die Rücklaufquote und das antwortende Kollektiv (Überrepräsentation von Ober- und Chefärzten) stets zu berücksichtigen, dass sich die Aussagen nur auf das untersuchte Kollektiv beziehen können und somit keine Allgemeingültigkeit abgeleitet werden kann.

Der Fragebogen ist der Versuch, eine komplexe Thematik und schwierig zu messende Größe, wie die subjektive Einschätzung der Patientensicherheit bzw. Aspekte davon, zu messen. Eine Landmarkstudie zu dieser Thematik mit ähnlichen Elementen ist die Studie von Singer et al. [23].

\section{Sicherheitskultur}

Die Sicherheitskultur in der eigenen Abteilung für Anästhesie wird von den Teilnehmern als deutlich positiver beschrieben als für das Krankenhaus insgesamt. Aus den erhobenen Daten geht jedoch nicht hervor, warum viele Umfrageteilnehmer dies so bewerten. Als Gründe kommen der mögliche, im Vergleich zur eigenen Abteilung nichtumfassende Überblick über die Maßnahmen zur Verbesserung der Sicherheitskultur anderer Abteilungen und die höhere Identifikation und damit evtl. bessere Bewertung der eigenen Abteilung infrage. $\mathrm{Da}$ die Anästhesie als Fachrichtung wie wenig andere Fachdisziplinen mit Sicherheitsaspekten und Quervergleichen zur Luftfahrt assoziiert wird (Stichwort "Cockpit-Strategie“[21]), mag das Thema Sicherheitskultur in diesem Fachgebiet einen vergleichsweise höheren Stellenwert haben. Dass die Sicherheitskultur in der eigenen Abteilung von jedem vierten Befragten und 


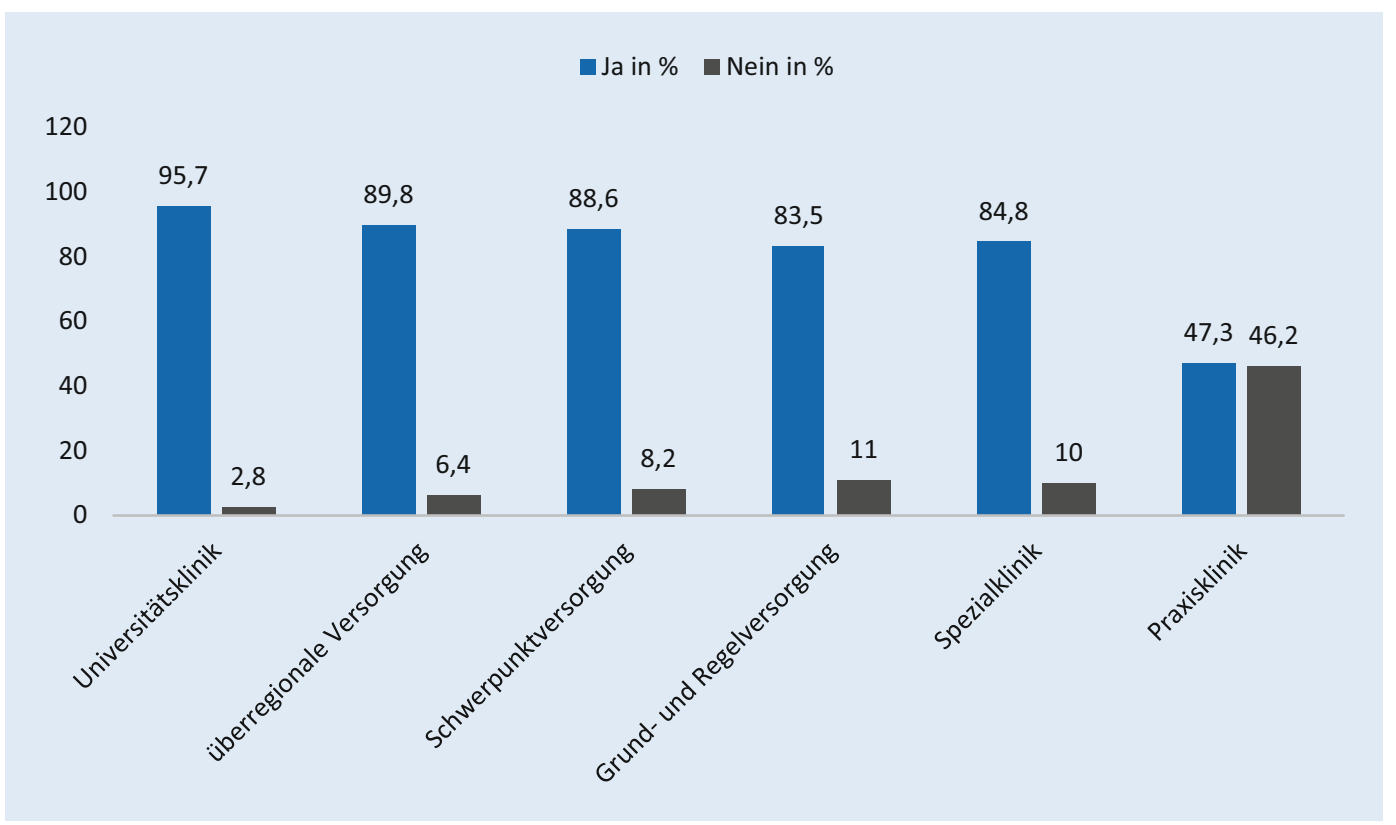

Abb. $1 \varangle$ Vorhandensein von CIRS in Abhängigkeit von der Versorgungsstufe des Krankenhauses. Fehlende Werte auf $100 \% \hat{=}$ "nicht bekannt"

für das gesamte Krankenhaus von fast jedem zweiten als negativ empfunden wird, zeigt, dass es in diesem Gebiet noch Verbesserungsbedarf gibt. Noch alarmierender ist die Tatsache, dass fast jeder vierte Befragte berichtet, dass die betreffende Person im Falle von unterlaufenen Fehlern bloßgestellt würde. Interessant ist die Tatsache, dass es in Bezug auf die Wahrnehmung von Situationen mit Bloßstellen im Rahmen von unterlaufenen Fehlern eine deutliche Diskrepanz zwischen Leitungspersonal (Chef- und Oberärzten) und Assistenz- und Fachärzten in unserem untersuchten Kollektiv vorzuherrschen scheint. Wenn Verhaltensweisen wie Bloßstellen annähernd häufig, wie hier berichtet, zum Alltag gehören sollten, wäre dies ein gravierender Missstand, der gesonderter Untersuchung bedürfte. Ebenso alarmierend ist, wenn jeder Fünfte berichtet, dass ein unterlaufener Fehler lieber verheimlicht würde. Auch hier zeigte sich, dass dies, je geringer die Stellung in der Hierarchiestufe war, desto häufiger angegeben wurde. Dies deutet darauf hin, dass in einer relevanten Zahl von Anästhesiekliniken in Deutschland der Umgang mit Fehlern als problematisch angesehen wird.

Auch eine eindeutig nichtflächendeckende Etablierung von Morbiditäts- und Mortalitätskonferenzen zur Aufarbeitung kritischer Patientenverläufe, wie hier gezeigt werden konnte, erscheint ohne Frage verbesserungsbedürftig. Dieses ist der
Patientensicherheit und Sicherheitskultur nicht zuträglich.

\section{Critical Incident Reporting System}

Annähernd jedem Anästhesisten ist das Akronym CIRS bekannt. In einer großen Mehrzahl der Kliniken ist CIRS zumindest formal etabliert. Etwas mehr als die Hälfte der antwortenden Anästhesisten haben bereits eine Meldung abgegeben. Dies ist als positiv zu werten. Die Krankenhäuser scheinen hier überwiegend ihrer gesetzlichen Pflicht nachzukommen. Eine Ausnahme bilden hier die Praxiskliniken - hier ist nur in etwa der Hälfte ein CIRS etabliert. Der ambulante Sektor scheint hier also einen großen Nachholbedarf zumindest in diesem Teilaspekt der Patientensicherheit und Sicherheitskultur aufzuweisen. Die Einschätzung bezüglich der Intensität der Nutzung in den Anästhesieabteilungen ist laut den befragten Teilnehmern heterogen. Laut jedem fünften Befragten wird CIRS fast gar nicht bzw. gar nicht verwendet.

Während die allermeisten Befragten angeben, dass CIRS-Meldungen anonym bleiben, verwundert, dass in fast einem Drittel der Fälle ein CIRS-Beauftragter der Anästhesieabteilung selbst oder gar der Chefarzt für Anästhesie erster Empfänger einer CIRS-Meldung ist. Nach dem NPSA London Protocol für eine Systemanalyse klinischer Zwischenfälle besteht ein Unter- sucherteam idealerweise aus einem Experten für Zwischenfallanalyse, einer externen Expertenmeinung, Expertise aus dem oberen Management, Expertise aus der klinischen Führungsebene sowie einer Person, welche die betroffene Station oder Abteilung gut kennt [13]. Laut dem Aktionsbündnis Patientensicherheit, welches umfangreiche Handlungsempfehlungen für die „Einrichtung und einen erfolgreichen Betrieb eines Berichts- und Lernsystems (CIRS)" veröffentlicht hat, ist ein zentraler und unabhängiger Anonymisierer sinnvoll [12]. Wenn ein Chefarzt erster Empfänger (womöglich sogar einziger Empfänger) einer CIRS-Meldung ist, könnte dies demgegenüber negative Folgen haben:

- Die Hemmschwelle etwas zu melden, könnte steigen.

- Beinahefehler oder kritische Verläufe, die durch organisatorische Schwächen oder Versäumnisse des Chefarztes selbst hervorgerufen sind (z. B. Pflegekraft meldet, dass ein Chefarzt Assistenzärzten beibringt, einen zentralen Venenkatheter anzulegen, ohne einen sterilen Kittel zu tragen), würden so kaum Gegenstand von CIRS-Meldungen, geschweige denn Änderungen der bisherigen Praxis.

- Selbstreferenz ohne externe Expertise.

- Anonymität gefährdet, wenn Meldung nicht zuvor anonymisiert und gefiltert wurde. 
Es wäre wünschenswert und für die Akzeptanz sowie das Funktionieren des CIRS von Vorteil, wenn, wie von über $42 \%$ der Befragten berichtet, ein übergeordneter CIRS-Beauftragter erster Empfänger der CIRS-Meldung wäre.

Es ist eine Forderung des Aktionsbündnis Patientensicherheit, dass die Mitarbeiter über die gemeldeten Ereignisse und die umgesetzten Maßnahmen regelmäßig Feedback erhalten. Insofern ist es überraschend und als kritisch zu werten, dass weniger als jeder dritte Teilnehmer berichten, regelmäßiges Feedback über CIRSMeldungen zu erhalten. Ein regelmäßiges Feedback zur Steigerung der Qualität von CIRS in vielen Krankenhäusern erscheint nur mangelhaft etabliert zu sein. Ebenso als kritisch zu werten ist die Tatsache, dass etwa ein Drittel der Antwortenden noch nicht erlebt hat, dass aus einer Meldung Konsequenzen gezogen worden sind.

Die Ergebnisse zeigen, dass ansonsten überwiegend Akzeptanz gegenüber dem CIRS besteht und es als lehrreich und wirksam empfunden wird. Umso mehr ist es wichtig, Feedbacksysteme zu etablieren. um den Beteiligten Probleme aufzuzeigen und gleichermaßen Lösungen an die Hand zu geben.

Situation, Background, Assessment, Recommendation System

Laut Beschluss des engeren Präsidiums der DGAl vom November 2015 wird die Verwendung von SBAR als strukturiertes Übergabeprotokoll im Sinne einer SOP empfohlen [3].

Zum Zeitpunkt der Befragung, 15 Monate nach dem Beschluss der DGAI, war etwa $70 \%$ der Befragten das Akronym SBAR nicht bekannt. Weniger als jeder zwölfte Befragte gab an, SBAR zu verwenden. Auf der anderen Seite scheinen die antwortenden Teilnehmer in der überwiegenden Mehrzahl einen großen Bedarf zu sehen, ein Hilfsmittel zur verbesserten Übergabequalität zur Hand zu haben. Die Tatsache, dass das SBAR-Konzept zum Umfragezeitpunkt nur eine marginale Durchdringung aufweist, mag an der fehlenden Eingängigkeit und etwas sperrigen, spiegelstrich- bzw. checklistenartigen Darstellung in oben genannten Publikation liegen. Ein intuitiveres Hilfsmittel zur verbes- serten Übergabequalität in Anlehnung an das SBAR-Konzept könnte hilfreich sein, die Durchdringung und Akzeptanz zu steigern. Dabei sollte dieses Hilfsmittel auch darauf abzielen, die Rahmenbedingungen der Patientenübergabe im Sinne einer konzentrierteren und ruhigeren Atmosphäre zu verbessern: So berichtet eine überwiegende Mehrzahl der befragten Teilnehmer, dass potenziell störende Handlungen am Patienten während der Übergaben stattfinden. Dieses könnte aufgrund nichtvollständiger Fokussierung auf die Entgegennahme von wichtigen Informationen zum gefährdenden Informationsverlust führen. Aus zahlreichen publizierten Untersuchungen geht hervor, dass sich die Übergaben ganz generell durch die Anwendung von Übergabe-Tools oder postoperativen Übergabechecklisten signifikant im Hinblick auf Vollständigkeit, Inhalt und Struktur verbessern [10, 14, 16, 20].

Diese Umfrage bietet, unter Berücksichtigung der Limitationen der niedrigen Rücklaufquote und der Überrepräsentation von Chef- und Oberärzten im Kollektiv der Antwortenden, erstmals ein Bild der empfundenen Sicherheitskultur und $\mathrm{Pa}$ tientensicherheit von deutschsprachigen Anästhesisten. In der hiesigen Literatur befindet sich derzeit keine weitere Untersuchung zu diesem Thema. Die vorliegende Untersuchung wirft aber auch zahlreiche Fragen auf, die Ansatz für weitere Untersuchungen sein können:

Systematische Beobachtungsstudien vor Ort in den Krankenhäusern etwa könnten noch mehr Aufschluss über die unterschiedlichen Teilaspekte der Sicherheitskultur von einzelnen Abteilungen und der gesamten Klinik sowie der Qualität der Patientensicherheit geben. Derartige Untersuchungen wären logistisch und vom Aufwand her sicher eine große Herausforderung.

Limitationen dieser Studie sind die Rücklaufquote und die Tatsache, dass Ober- und Chefärzte im antwortenden Kollektiv mit $45 \%$ deutlich überrepräsentiert sind. Aussagen können sich also, wie es ganz generell der Fall in Umfragen ist, nur auf das untersuchte Studienkollektiv beziehen. Umfragen mit einer niedrigen Rücklaufquote und einem nicht der im Krankenhaus entsprechenden Verteilung von Chef-, Ober- und Assistenzärzten kön- nen einem erhöhten Bias unterliegen. Bei der relevanten Anzahl von Antwortenden im vierstelligen Bereich lassen sich jedoch durchaus allgemeingültige Aussagetrends feststellen.

- Die Sicherheitskultur in der eigenen Abteilung wird von jedem vierten Befragten und die Sicherheitskultur im gesamten Krankenhaus von fast jedem zweiten als negativ empfunden. Dies deutet auf eine Unzufriedenheit der befragten Anästhesisten bei diesem Thema hin. Eine flächendeckendere Etablierung von Morbiditäts- und Mortalitätskonferenzen zur Aufarbeitung kritischer Patientenverläufe erscheint im Sinne der Steigerung der Patientensicherheit notwendig. In einer relevanten Zahl von Abteilungen für Anästhesie scheint noch die Unkultur des Bloßstellens nach individuellen Fehlern einer Atmosphäre für eine gute Fehlerkultur entgegenzustehen.

- CIRS ist in einer großen Mehrzahl der Kliniken formal etabliert. Die Anwendung des CIRS scheint aber in vielen Punkten verbesserungswürdig: Der Grad der Nutzung scheint nicht ausgeschöpft zu sein. In vielen Fällen ist der erste Empfänger der Meldung ein Mitarbeiter der Klinik und kein übergeordneter CIRS-Beauftragter. Das Aktionsbündnis Patientensicherheit fordert regelmäßiges Feedback dieses scheint im deutschsprachigen Raum unzureichend etabliert zu sein.

- Der Beschluss des engeren Präsidiums der DGAI, das SBAR-System zur verbesserten Übergabe zu verwenden, ist nicht etabliert. Es besteht der Wunsch nach einem geeigneten Hilfsmittel zur Verbesserung der Patientenübergabe. Die überwiegende Mehrzahl an befragten Anästhesisten beobachtet, dass während Patientenübergaben Parallelhandlungen am Patienten durchgeführt werden. Dies kann zu gefährlichem Informationsverlust führen.

9 Kernaussagen:

- Wichtige Aspekte der Sicherheitskultur in Anästhesieabteilungen werden von den Teilnehmern als deutlich positiver beschrieben als für das Krankenhaus insgesamt. 
- In einer relevanten Zahl (23\%) von Krankenhäusern wird die betreffende Person im Falle von unterlaufenen Fehlern bloßgestellt.

- Assistenz- und Fachärzte empfinden die Kommunikation über fehlerhafte Verläufe nicht so positiv wie Ober- und Fachärzte.

- Assistenz- und Fachärzte empfinden in einer relevanten Zahl und häufiger als Chef- und Oberärzte, dass im Falle von Fehlern Bloßstellungen stattfinden.

- Morbiditäts- und Mortalitätskonferenzen zum Aufarbeiten kritischer Patientenverläufe sind deutschlandweit nicht flächendeckend verbreitet.

- CIRS ist in der deutschen Krankenhauslandschaft weit verbreitet - dies gilt jedoch nicht für Praxiskliniken.

- Ein regelmäßiges Feedback zu den abgegebenen CIRS-Meldungen zur Steigerung der Qualität von CIRS scheint nur mangelhaft etabliert zu sein. Dies sollte sich ändern.

- Der Beschluss des engeren Präsidiums der DGAI vom November 2015 die Verwendung von SBAR als strukturiertes Übergabeprotokoll im Sinne einer SOP zu empfehlen ist nicht annähernd etabliert. 15 Monate nach Beschlussfassung war SBAR $70 \%$ der befragten Anästhesisten unbekannt.

- Eine große Mehrheit der befragten Teilnehmer wünscht sich ein Hilfsmittel oder eine bestimmte Struktur, um die eigene Patientenübergabe $z u$ verbessern.

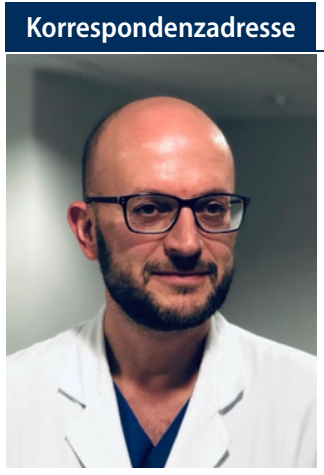

Dr. med. Hanke E. Marcus, DESA

Klinik für Anästhesiologie und operative Intensivmedizin, Klinikum der Universität Witten/Herdecke, Kliniken der Stadt Köln Köln, Deutschland marcush@kliniken-koeln.de
Funding. Open Access funding enabled and organized by Projekt DEAL.

\section{Einhaltung ethischer Richtlinien}

Interessenkonflikt. H.E. Marcus, W. Golbeck, M. Schieren, J. Defosse, F. Wappler und A. Böhmer geben an, dass kein Interessenkonflikt besteht.

Für diesen Beitrag wurden von den Autoren keine Studien an Menschen oder Tieren durchgeführt. Für die aufgeführten Studien gelten die jeweils dort angegebenen ethischen Richtlinien.

Open Access. Dieser Artikel wird unter der Creative Commons Namensnennung 4.0 International Lizenz veröffentlicht, welche die Nutzung, Vervielfältigung, Bearbeitung, Verbreitung und Wiedergabe in jeglichem Medium und Format erlaubt, sofern Sie den/die ursprünglichen Autor(en) und die Quelle ordnungsgemäß nennen, einen Link zur Creative Commons Lizenz beifügen und angeben, ob Änderungen vorgenommen wurden.

Die in diesem Artikel enthaltenen Bilder und sonstiges Drittmaterial unterliegen ebenfalls der genannten Creative Commons Lizenz, sofern sich aus der Abbildungslegende nichts anderes ergibt. Sofern das betreffende Material nicht unter der genannten Creative Commons Lizenz steht und die betreffende Handlung nicht nach gesetzlichen Vorschriften erlaubt ist, ist für die oben aufgeführten Weiterverwendungen des Materials die Einwilligung des jeweiligen Rechteinhabers einzuholen.

Weitere Details zur Lizenz entnehmen Sie bitte der Lizenzinformation auf http://creativecommons.org/ licenses/by/4.0/deed.de.

\section{Literatur}

\section{Verwendete Literatur}

1. Colla JB, Bracken AC, Kinney LM, Weeks WB (2005) Measuring patient safety climate: a review of surveys. Qual Saf Health Care 14(5):364-366

2. Das Patientenrechtegesetz, der Gemeinsame Bundesausschuss und CIRS. BDAktuell. DGAInfo. https://www.cirs-ains.de/files/fachpresse/AI_ 09-2014_Verbaende_BDA-DGAI_CIRS_Neu.pdf. Zugegriffen: 8.Dez. 2019

3. DGAI Info (2016) Empfehlung. Strukturierte Patientenübergabe in der perioperativen Phase. Das SBAR-Konzept. Anasth Intensivmed 57:88-90

4. Eichhorn JH (1989) Prevention of intraoperative anesthesia accidents and related severe injury through safety monitoring. Anesthesiology 70(4):572-577

5. Flin R, Yule S, McKenzie L, Paterson-Brown S, Maran N (2006) Attitudes to teamworkand safety in the operating theatre. Surgeon 4(3):145-151

6. Gemeinsamer Bundesausschuss, Richtlinie Qualitätsmanagement - Richtlinie Krankenhäuser, Stand: 23. Januar 2014 des Gemeinsamen Bundesausschusses über die grundsätzlichen Anforderungen an ein einrichtungsinternes Qualitätsmanagementfürnach §108SGBV zugelassene Krankenhäuser (Qualitätsmanagement - Richtlinie Krankenhäuser - KQM - RL), im Internet unter https://www.g-ba.de/downloads/62-492-
865/KQM-RL_2014-01-23.pdf (zuletzt aufgerufen 8. Dez. 2019)

7. Gemeinsamer Bundesausschuss, Tragende Gründe zum Beschluss des Gemeinsamen Bundesausschusses über eine Änderung der Vereinbarung des Gemeinsamen Bundesausschusses gemäß § 137 Abs. 1 Satz 3 Nr. 1 SGBV über die grundsätzlichen Anforderungen an ein einrichtungsinternes Qualitätsmanagement für nach § 108 SGB V zugelassene Krankenhäuser: Umsetzung des § 137 Absatz 1d Satz 1 SGB V, Stand: 23. Januar 2014; im Internet unter: https://www.g-ba.de/downloads/ 40-268-2709/2014-01-23_KQM-RL_137-1d_TrG. pdf (zuletzt aufgerufen 8. Dez. 2019)

8. Gemeinsamer Bundesausschuss Tragende Gründe zum Beschlussentwurf des Gemeinsamen Bundesausschusses über eine Änderung der Qualitätsmanagement-Richtlinie vertragsärztlicher Versorgung: Umsetzung des $\S 137$ Absatz 1d Satz 1 SGB V, Stand: 23. Januar 2014. https:// www.g-ba.de/downloads/40-268-2667/201401-23_AEQM-RL_Umsetzung-137-1d_TrG.pdf. Zugegriffen:08.12.2019

9. Guldenmund FW (2000) The nature of safety culture: a review of theory and research. Saf Sci 34(1-3):215-257

10. Hall AJ, Toner NS, Bhatt PM (2019) The introduction of a neurosurgical postoperative checklist improved quality of care and patient safety. $\mathrm{Br} \mathrm{J}$ Neurosurg 22:1-5

11. http://www.institut-schreier.de/files/Analyse_JaProzente.pdf.Zugegriffen:8.Dez. 2019

12. https://www.aps-ev.de/wp-content/uploads/ 2016/10/160913_CIRS-Broschuere_WEB.pdf.Zugegriffen: 8.Dez. 2019

13. https://www.imperial.ac.uk/media/imperialcollege/medicine/surgery-cancer/pstrc/ londonprotocol_e.pdf. Zugegriffen: 8. Dez. 2019

14. Marshall AP, Tobiano G, Murphy N et al (2019) Handover from operating theatre to the intensive care unit: a quality improvement study. Aust Crit Care 32(3):229-236

15. Marx GF, Mateo CV, Orkin LR (1973) Computer analysis of postanesthetic deaths. Anesthesiology 39(1):54-58

16. Redley B, Bucknall TK, Evans S, Botti M (2016) Interprofessional clinical handover in post-anaesthetic care units: tools to improve quality and safety. Int J Qual Health Care 28(5):573-579

17. Schieren M, Böhmer A, Golbeck W, Defosse J, Wappler F, Marcus HE (2018) Anesthesia problem cards-indispensable yet problematic : nationwide survey on experiences from clinical practice. Anaesthesist 67(4):264-269

18. Sexton JB, Thomas EJ, Helmreich RL (2000) Error, stress and teamwork in medicine and aviation. A cross-sectional study. Chirurg 71(6 suppl):138-142

19. Singla AK, Kitch BT, Weissman JS, Campbell EG (2006) Assessing patient safety culture: a review and synthesis of the measurement tools. J Patient Saf 2:105-115

20. Tun KS, Wai KS, Yin Y, Thein MK (2019) Postoperative handover among nurses in an orthopedic surgical setting in Myanmar: a best practice implementation project. JBI Database System Rev Implement Rep. https://doi.org/10.11124/ JBISRIR-2017-004015

21. Vogelsang $H$, Botteck NM, Herzog-Niescery J et al (2019) Transfer of a cockpit strategy to anesthesiology: clinical example: introduction of canned decisions to solve cannot intubate cannot oxygenate situations. Anaesthesist 68(1):30-38 
22. www.jointcommission.org/assets/1/6/2007_Annual_Report.pdf.Zugegriffen:8.Dez. 2019

23. Singer SJ, Gaba DM, Geppert JJ, Sinaiko AD, Howard SK, Park KC (2003) The culture of safety: results of an organization-wide survey in 15 California hospitals. Qual Saf Health Care 12(2):112-118

24. Wollner E, Nourian MM, Booth W, Conover S, Law T, Lilaonitkul M, Gelb AW, LipnickMS (2020) Impact of capnography on patient safety in high- and lowincome settings: a scoping review. $\mathrm{Br} J$ Anaesth 125(1):e88-e103

\section{Weiterführende Literatur}

25. Lagasse RS (2002) Anesthesia safety: model or myth? A review of the published literature and analysis of current original data. Anesthesiology 97(6):1609-1617

\section{On the safe side? Safety culture and patient safety in German anesthesiology clinics-A nationwide survey on the status quo}

Background: A good safety culture may be predominantly defined by an open and unsanctioned communication about critical and erroneous courses. In an effort to improve patient safety various instruments, such as the critical incident reporting system (CIRS) or in terms of patient handover, the use of the situation, background, assessment, recommendation (SBAR) system patient handover, have been developed and are recommended by the German Society of Anaesthesiology and Intensive Care Medicine (DGAI). This study aimed at identifying how anesthesiologists perceive the safety culture in their current department and whether CIRS or SBAR are already established or not.

Material and methods: All registered members of the DGAI and the Professional Association of German Anaesthesiologists (BDA, $n=19,042$ ) were invited to participate in an online survey on patient safety. In this survey there was a focus on the perceived safety culture and the experience with CIRS and SBAR.

Results: Of the participants $76.6 \%(n=1372)$ stated that their department of anesthesiology has a good safety culture, while in $23.4 \%(n=419)$ there was not. For the whole hospital the safety culture was only rated as being positive by $54.3 \%$ $(n=949)$ of the respondents. An open communication about critical and erroneous courses occurred in $76.5 \%(n=1375)$ according to the participants, $23.0 \%(n=408)$ had the impression that in the case of errors the respective person was being denounced. In one third of the participants' departments $(n=630,36.6 \%)$ there were no morbidity and mortality conferences. The acronym CIRS was familiar to $98.9 \%(n=1801)$ of the participants, $84.8 \%(n=1544)$ of the surveyed anesthesiologists reported that CIRS was established in their departments. Critical incidents have been reported via CIRS by $54.4 \%$ ( $n=839)$ of the respondents. Only $29.4 \%(n=462)$ of the participants received regular feedback on CIRS reports. The acronym SBAR as a handover tool is unknown to the majority of the surveyed participants $(n=1181,63.7 \%)$ and $86.1 \%(n=1554)$ consider using an instrument in order to improve handover quality as possibly being beneficial.

Conclusion: Anesthesiologists rate the quality of the safety culture of their own anesthesiology department to be higher compared to their hospital in general. In some hospitals there is denouncement in cases of erroneous courses according to the respondents. In the patients' point of view morbidity and mortality conferences should be established more often. CIRS is known to almost every surveyed anesthetist but feedback on a regular basis is sparse. This contradicts the claims of the German Coalition for Patient Safety. The acronym SBAR is unknown to the majority of surveyed participants despite the recommendation of the DGAI to implement it. There is a wish for tools in order to enhance the quality of handovers.

\section{Keywords}

Patient safety · Safety culture · Critical incident reporting system · Patient handover $\cdot$ Survey 